\title{
Solution of Two-Dimensional Riemann Problems for Gas Dynamics without Riemann Problem Solvers
}

\author{
Alexander Kurganov, ${ }^{1, *}$ Eitan Tadmor ${ }^{2}$ \\ ${ }^{1}$ Department of Mathematics, University of Michigan, Ann Arbor, Michigan 48109 \\ ${ }^{2}$ Department of Mathematics, UCLA, Los Angeles, California 90095
}

Received 10 January 2002; accepted 28 January 2002

We report here on our numerical study of the two-dimensional Riemann problem for the compressible Euler equations. Compared with the relatively simple 1-D configurations, the 2-D case consists of a plethora of geometric wave patterns that pose a computational challenge for high-resolution methods. The main feature in the present computations of these 2-D waves is the use of the Riemann-solvers-free central schemes presented by Kurganov et al. This family of central schemes avoids the intricate and timeconsuming computation of the eigensystem of the problem and hence offers a considerably simpler alternative to upwind methods. The numerical results illustrate that despite their simplicity, the central schemes are able to recover with comparable high resolution, the various features observed in the earlier, more expensive computations. ๑ 2002 Wiley Periodicals, Inc. Numer Methods Partial Differential Eq 18: $584-608,2002$

Keywords: multidimensional conservation laws; Euler equations of gas dynamics; Riemann problem; semi-discrete central schemes; nonoscillatory piecewise polynomial reconstructions

\section{INTRODUCTION}

We report here on our numerical study of two-dimensional (2-D) Riemann problem for the compressible Euler equations, following the works of Schultz-Rinne et al. [1, 2], Chang et al. [3], Zhang and Zheng [4], Lax and Liu [5], and Chang et al. [6].

Correspondence to: E. Tadmor, Department of Mathematics, University of California, Los Angeles, 405 H-lgard Avenue, Los Angeles, CA 90095-1555 (e-mail: tadmor@math.ucla.edu)

AMS subject classification: Primary 65M10; Secondary 65M05

*Present address: Department of Mathematics, Tulane University, New Orleans, LA 70118 (e-mail: kurganov@math.lsa.umich.edu

Contract grant sponsor: National Science Foundation Group Infrastructure Grant (A.K.)

Contract grant sponsor: National Science Foundation (A.K.); contract grant number: DMS00-73631

Contract grant sponsor: ONR (E.T.); contract grant number: N0014-91-J-1076

Contract grant sponsor: National Science Foundation (E.T.); contract grant number: DMS01-07428 and DMS01-07917

(๑) 2002 Wiley Periodicals, Inc. 
Before turning to the 2-D case, we recall the corresponding 1-D setup. The 1-D Riemann problem could be solved in terms of a succession of centered waves [7]. In particular, the 1-D centered waves associated with gas dynamics equations consist of shock-, rarefaction-, and contact-waves, [7-9]. The exact (or approximate) 1-D Riemann problem solvers serve as a building block for the large class of so-called upwind schemes, following the seminal work of Godunov [8]. The other class of so-called central schemes offers an alternative to upwind methods by avoiding the time-consuming computation of (approximate) Riemann problem solvers, yet retaining the desired high resolution. Unlike the 1-D case, however, no explicit Riemann solvers are available in the 2-D case. Indeed, the 2-D Riemann problem separated by 1-D elementary waves offers a plethora of no less than 19 different admissible configurations [1-6], which therefore cannot be used as a building block in the 2-D case. Consequently, 2-D upwind schemes require some sort of dimensional splitting, where 1-D Riemann problems are solved, one dimension at the time. The advantage of the Riemann-solvers-free central schemes is therefore further amplified in the 2-D case. By avoiding the intricate and time-consuming computation of the eigen structures in 1-D and in particular, 2-D problems, we end up with a considerably simpler and faster class of high-resolution schemes.

Central schemes can be formulated along the lines of the original Godunov's framework [8], namely, realizing the evolution of piecewise polynomial solution after each small time step by its cell averages. To avoid Riemann problem solvers, however, the solution of central schemes is realized by cell averages computed over staggered cells, which in turn yield numerical fluxes located inside the smooth part of the piecewise solution. In the original 1-D second-order central scheme of Nessyahu and Tadmor [10], and its higher-order and 2-D generalizations [11, 12], cells of typical spatial length $\Delta x$ were staggered in alternate time steps, by being placed $\Delta x / 2$ away from each other. A survey on this class of central schemes can be found in the C.I.M.E. Lectures Notes [13, pp. 47-82]. In the more recent, less dissipative versions of central schemes presented in [14-18], staggered cells were placed in a distance of order $\mathcal{O}(\Delta t)$ from each other. The latter versions admit a particularly simple semi-discrete limit by letting $\Delta t \downarrow 0$. Consequently, alternating cells collapse onto each other in the semi-discrete limit and staggering is avoided altogether. Let us also mention that there are other derivations of central schemes that lack any specific interpratation as Godunov/Finite-Volume methods. Most notably, central schemes could be derived as zero-relaxation limits for a proper subclass of the relaxation methods presented in [19], or, by combining componenetwise ENO/WENO reconstructions together with flux splitting advocated in [20].

In Section 2 we provide a brief description of the central schemes proposed in [17], which have been applied to the 2-D Euler equations of gas dynamics in Section 3. Compared with the "simple" 1-D configurations, the 2-D case offers 19 different configurations which consist of a considerably richer variety of 2-D geometric patterns formed by shocks, rarefactions, slip lines, and contacts. The main feature of the present computation is the use of Riemann-solvers-free central schemes to resolve this variety of wave formations. Remarkably, the numerical results reported in Section 3 show that despite the lack of any specific "physical" input beyond the maximal local speeds, the central schemes recover with a comparable high-resolution, all the features observed by the earlier, more expensive computations based on upwind schemes.

\section{GENUINELY MULTIDIMENSIONAL SEMI-DISCRETE CENTRAL SCHEMES 2.1. Fully Discrete Central Schemes}

We consider a general two-dimensional system of hyperbolic conservation laws,

$$
u_{t}+f(u)_{x}+g(u)_{y}=0 \text {. }
$$




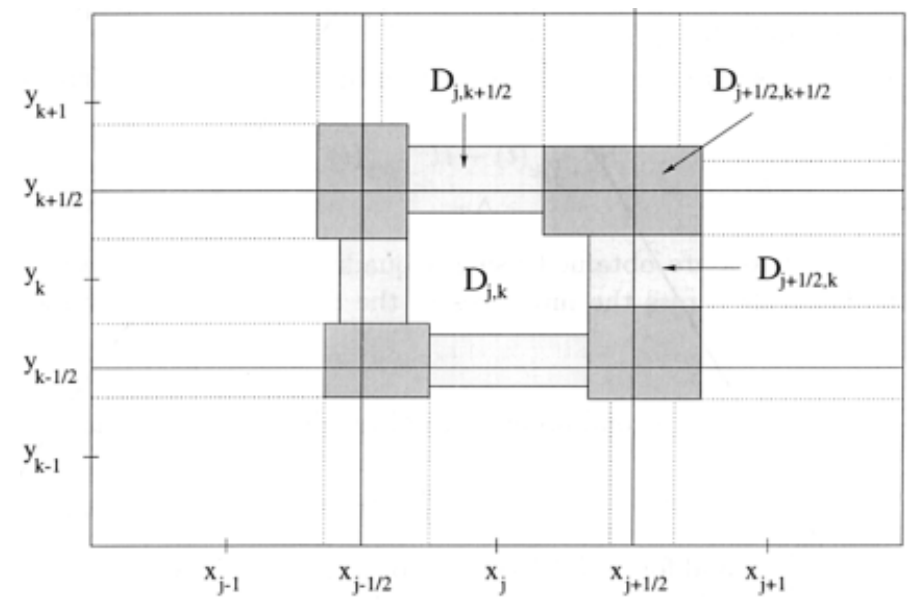

FIG. 2.1. Two-dimensional central-upwind differencing.

The computed solution is realized in terms of the cell averages

$$
\bar{u}_{j, k}^{n}:=\frac{1}{\Delta x \Delta y} \int_{x_{j-1 / 2}}^{x_{j+1 / 2}} \int_{y_{k-1 / 2}}^{y_{k+1 / 2}} u\left(x, y, t^{n}\right) d x d y,
$$

based on spatial cells $I_{j k}=\left[x_{j-1 / 2}, x_{j+1 / 2}\right] \times\left[y_{k-1 / 2}, y_{k+1 / 2}\right]$. Here and below, $\left(x_{\alpha}, y_{\beta}\right)=(\alpha \Delta x$, $\beta \Delta y$ ) denote the coordinates of the computational grid. To advance the computation to the next time level at $t=t^{n+1}$, we proceed with three steps of reconstruction, evolution, and projection.

Starting with the given cell averages $\bar{u}_{j, k}^{n}$, the first step consists of reconstructing a nonoscillatory piecewise polynomial of the form

$$
\tilde{u}^{n}(x, y):=\sum_{j, k} p_{j, k}^{n}(x, y) \chi_{I_{j k}}(x, y)
$$

where the $\chi$ 's are the characteristic functions of the corresponding intervals. Different choices of polynomial reconstructions result in different types of central schemes. Few choices will be outlined below in (2.5), (2.6). In the second step, we evolve the piecewise polynomial $\tilde{u}^{n}(x, y)$ in time by solving the initial-value problem (2.1), (2.2). Each of the polynomial pieces of $\tilde{u}^{n}(x$, $y$ ) centered around the vertices $\left(x_{j \pm 1 / 2}, y_{k \pm 1 / 2}\right)$ is propagated within a "rectangular cone" of influence, $D_{j \pm 1 / 2, k \pm 1 / 2}$, whose boundaries propagate with different right- and left-sided local speeds, consult the floor plan in Figure 2.1. The computed values of the local speeds $a_{j+1 / 2, k}^{ \pm}$, $b_{j, k+1 / 2}^{ \pm}$are specified below at (2.8).

Integrating (2.1), (2.2) over rectangular control volumes erected under the aforementioned domains, $D_{\alpha \beta} \times\left[t^{n}, t^{n+1}\right]$, results in the new cell averages at time $t=t^{n+1}$, which are denoted, respectively, by $\left\{\bar{w}_{j, k+1 / 2}^{n+1}\right\},\left\{\bar{w}_{j+1 / 2, k}^{n+1}\right\},\left\{\bar{w}_{j+1 / 2, k+1 / 2}^{n+1}\right\}$, and $\left\{\bar{w}_{j, k}^{n+1}\right\}$. These cell averages can be computed explicitly following the approach in [12], using appropriate quadrature rules to approximate the flux across the temporal interfaces (consult [17] for details). Next, the new cell averages, $\left\{\bar{w}_{\alpha, \beta}^{n+1}\right\}$ are used to reconstruct new nonoscillatory polynomials, $\left\{\tilde{w}_{\alpha, \beta}^{n+1}(x, y)\right\}$, and at this stage we end up with an approximate piecewise polynomial solution at $t=t^{n+1}$ of the form 


$$
\begin{aligned}
\tilde{w}^{n+1}(x, y):=\sum_{j, k}\left[\tilde{w}_{j, k}^{n+1}(x, y) \chi_{D_{j k}}(x, y)+\tilde{w}_{j+1 / 2, k}^{n+1}(x, y) \chi_{D_{j+1 / 2, k}}(x, y)\right. & +\tilde{w}_{j, k+1 / 2}^{n+1}(x, y) \chi_{D_{j, k+1 / 2}}(x, y) \\
& \left.+\tilde{w}_{j+1 / 2, k+1 / 2}^{n+1}(x, y) \chi_{D_{j+1 / 2, k+1 / 2}}(x, y)\right] .
\end{aligned}
$$

Finally, we conclude by projecting this computed solution back onto the original cells, which is again realized in terms of the cell averages

$$
\bar{u}_{j, k}^{n+1}=\frac{1}{\Delta x \Delta y} \int_{x_{j-1 / 2}}^{x_{j+1 / 2}} \int_{y_{k-1 / 2}}^{y_{k+1 / 2}} \tilde{w}^{n+1}(x, y) d x d y .
$$

The above derivation results in the second- or third-order fully-discrete central schemes, with explicit yet complicated formulae. A particular advantage of this type of central schemes, compared with the original staggered version of central schemes introduced in [12], is the simplification that could be achieved by taking a semi-discrete limit, letting $\Delta t \downarrow 0$.

\subsection{The Semi-Discrete Limit}

Following the approach in $[14,16,17]$, we consider the central algorithm described above and pass to the limit as $\Delta t \rightarrow 0$. Notice that the cone of influence, $D_{j k} \times\left[t^{n}, t^{n}+\Delta t\right]$, falls back onto the original cell, $I_{j k}$, we have started with at $t=t^{n}$.

The resulting semi-discrete scheme can be written in the conservative form (see [17] for the detailed derivation),

$$
\frac{d}{d t} \bar{u}_{j, k}(t)=-\frac{H_{j+1 / 2, k}^{x}(t)-H_{j-1 / 2, k}^{x}(t)}{\Delta x}-\frac{H_{j, k+1 / 2}^{y}(t)-H_{j, k-1 / 2}^{y}(t)}{\Delta y} .
$$

Here, the numerical fluxes are obtained using a quadrature formula of an appropriate order for approximating the integrals across the interfaces of the domains $D_{j \pm 1 / 2, k}$ and $D_{j, k \pm 1 / 2}$. We consider few examples.

A Second-order Method. A second-order method requires a piecewise linear reconstruction, (2.2), of the form

$$
p_{j, k}^{n}(x, y)=\bar{u}_{j, k}^{n}+\left(u_{x}\right)_{j, k}^{n}\left(x-x_{j}\right)+\left(u_{y}\right)_{j, k}^{n}\left(y-y_{k}\right)
$$

Here, $\left(u_{x}\right)_{j, k}^{n}$ and $\left(u_{y}\right)_{j, k}^{n}$ stand for an (at least first-order) approximation to the derivatives $u_{x}\left(x_{j}\right.$, $\left.y_{k}, t^{n}\right)$ and $u_{y}\left(x_{j}, y_{k}, t^{n}\right)$, respectively. To ensure a nonoscillatory nature of the reconstruction (2.2)-(2.5), one needs to use a nonlinear limiter in the computation of these slopes. This can be done in many different ways (see, e.g., [21-24]). In this article, we have used van Leer's one-parameter family of the minmod limiters [25, 21, 24]

$$
\begin{aligned}
& \left(u_{x}\right)_{j, k}=\operatorname{minmod}\left(\theta \frac{\bar{u}_{j+1, k}-\bar{u}_{j, k}}{\Delta x}, \frac{\bar{u}_{j+1, k}-\bar{u}_{j-1, k}}{2 \Delta x}, \theta \frac{\bar{u}_{j, k}-\bar{u}_{j-1, k}}{\Delta x}\right), \\
& \left(u_{y}\right)_{j, k}=\operatorname{minmod}\left(\theta \frac{\bar{u}_{j, k+1}-\bar{u}_{j, k}}{\Delta y}, \frac{\bar{u}_{j, k+1}-\bar{u}_{j, k-1}}{2 \Delta y}, \theta \frac{\bar{u}_{j, k}-\bar{u}_{j, k-1}}{\Delta y}\right),
\end{aligned}
$$


where $\theta \in[1,2]$, and the multivariable minmod function is defined by

$$
\min \bmod \left(x_{1}, x_{2}, \ldots\right):= \begin{cases}\min _{j}\left\{x_{j}\right\}, & \text { if } x_{j}>0 \forall j \\ \max _{j}\left\{x_{j}\right\}, & \text { if } x_{j}<0 \forall j \\ 0, & \text { otherwise }\end{cases}
$$

Remark. Notice that in the scalar case, larger $\theta$ 's in (2.6) correspond to less dissipative, but still nonoscillatory limiters [12, 14, 16, 17]. For systems of conservation laws, no proof of a nonoscillatory property is available. Nevertheless, a large variety of computations performed with central schemes confirm stability and lack of spurious oscillations while achieving high resolution throughout the computational domain. In particular, central schemes owe their considerable simplicity to implementation of the minmod limiter (2.6) componentwise; no need for eigen decomposition of the vectors of divided differences. Our numerical experiments (Section 3, see also $[14,16,17]$ ) indicate that the optimal values of $\theta$ vary between 1 and 1.5.

Given the piecewise linear polynomial we can compute the reconstructed values at the interfaces

$$
u_{j, k}^{\mathrm{N}}:=p_{j, k}^{n}\left(x_{j}, y_{k+1 / 2}\right), u_{j, k}^{\mathrm{S}}:=p_{j, k}^{n}\left(x_{j}, y_{k-1 / 2}\right), u_{j, k}^{\mathrm{E}}:=p_{j, k}^{n}\left(x_{j+1 / 2}, y_{k}\right), u_{j, k}^{\mathrm{W}}:=p_{j, k}^{n}\left(x_{j-1 / 2}, y_{k}\right) .
$$

These interfaces are moving with the corresponding speeds

$$
\begin{aligned}
& a_{j+1 / 2, k}^{+}:=\max \left\{\lambda_{N}\left(\frac{\partial f}{\partial u}\left(u_{j+1, k}^{\mathrm{W}}\right)\right), \lambda_{N}\left(\frac{\partial f}{\partial u}\left(u_{j, k}^{\mathrm{E}}\right)\right), 0\right\}, \\
& b_{j, k+1 / 2}^{+}:=\max \left\{\lambda_{N}\left(\frac{\partial g}{\partial u}\left(u_{j, k+1}^{\mathrm{S}}\right)\right), \lambda_{N}\left(\frac{\partial g}{\partial u}\left(u_{j, k}^{\mathrm{N}}\right)\right), 0\right\}, \\
& a_{j+1 / 2, k}^{-}:=\min \left\{\lambda_{1}\left(\frac{\partial f}{\partial u}\left(u_{j+1, k}^{\mathrm{W}}\right)\right), \lambda_{1}\left(\frac{\partial f}{\partial u}\left(u_{j, k}^{\mathrm{E}}\right)\right), 0\right\}, \\
& b_{j, k+1 / 2}^{-}:=\min \left\{\lambda_{1}\left(\frac{\partial g}{\partial u}\left(u_{j, k+1}^{\mathrm{S}}\right)\right), \lambda_{1}\left(\frac{\partial g}{\partial u}\left(u_{j, k}^{\mathrm{N}}\right)\right), 0\right\},
\end{aligned}
$$

where $\lambda_{N}$ and $\lambda_{1}$ denote the largest and the smallest eigenvalues of the Jacobians $(\partial f / \partial u)$ and $(\partial g / \partial u)$, respectively.

Using second-order midpoint rule to approximate the spatial integrals along the faces of side cells, $D_{j+1 / 2, k}$ and $D_{j, k+1 / 2}$, results in the second-order numerical fluxes

$$
H_{j+1 / 2, k}^{x}=\frac{a_{j+1 / 2, k}^{+} f\left(u_{j, k}^{\mathrm{E}}\right)-a_{j+1 / 2, k}^{-} f\left(u_{j+1, k}^{\mathrm{W}}\right)}{a_{j+1 / 2, k}^{+}-a_{j+1 / 2, k}^{-}}+\frac{a_{j+1 / 2, k}^{+} a_{j+1 / 2, k}^{-}}{a_{j+1 / 2, k}^{+}-a_{j+1 / 2, k}^{-}}\left[u_{j+1, k}^{\mathrm{W}}-u_{j, k}^{\mathrm{E}}\right],
$$

and

$$
H_{j, k+1 / 2}^{y}=\frac{b_{j, k+1 / 2}^{+} g\left(u_{j, k}^{\mathrm{N}}\right)-b_{j, k+1 / 2}^{-} g\left(u_{j, k+1}^{\mathrm{S}}\right)}{b_{j, k+1 / 2}^{+}-b_{j, k+1 / 2}^{-}}+\frac{b_{j, k+1 / 2}^{+} b_{j, k+1 / 2}^{-}}{b_{j, k+1 / 2}^{+}-b_{j, k+1 / 2}^{-}}\left[u_{j, k+1}^{\mathrm{S}}-u_{j, k}^{\mathrm{N}}\right] .
$$


Remark. The computation in (2.8) takes into account the different local speeds from each side of the $x$ - and $y$-interfaces. If we further simplify and use a symmetric cone of propagation with local speeds $a_{j+1 / 2, k}^{ \pm}:= \pm \max \left\{\left|a_{j+1 / 2, k}^{+}\right|,\left|a_{j+1 / 2, k}^{-}\right|\right\}, b_{j, k+1 / 2}^{ \pm}:= \pm \max \left\{\left|b_{j, k+1 / 2}^{+}\right|,\left|b_{j, k+1 / 2}^{-}\right|\right\}$, then the central scheme (2.4), (2.9)-(2.10) is reduced to the central scheme introduced earlier in [14]. The refinement, introduced in [17], requires a more precise cone of propagation, which nevertheless avoids any additional information on the eigen structure of the problem.

An Alternative Second-order Method. With the same piecewise linear reconstruction as before, (2.5), we introduce the corner values

$$
u_{j, k}^{\mathrm{NE}(\mathrm{NW})}:=p_{j, k}^{n}\left(x_{j \pm 1 / 2}, y_{k+1 / 2}\right), u_{j, k}^{\mathrm{SE}(\mathrm{SW})}:=p_{j, k}^{n}\left(x_{j \pm 1 / 2}, y_{k-1 / 2}\right) .
$$

Replacing the second-order midpoint rule with the trapezoidal rule gives the alternative second-order numerical fluxes:

$$
\begin{aligned}
H_{j+1 / 2, k}^{x}:=\frac{a_{j+1 / 2, k}^{+}}{2\left(a_{j+1 / 2, k}^{+}-a_{j+1 / 2, k}^{-}\right)}\left[f\left(u_{j, k}^{\mathrm{NE}}\right)\right. & \left.+f\left(u_{j, k}^{\mathrm{SE}}\right)\right]-\frac{a_{j+1 / 2, k}^{-}}{2\left(a_{j+1 / 2, k}^{+}-a_{j+1 / 2, k}^{-}\right)}\left[f\left(u_{j+1, k}^{\mathrm{NW}}\right)+f\left(u_{j+1, k}^{\mathrm{SW}}\right)\right] \\
& +\frac{a_{j+1 / 2, k}^{+} a_{j+1 / 2, k}^{-}}{2\left(a_{j+1 / 2, k}^{+}-a_{j+1 / 2, k}^{-}\right)}\left[u_{j+1, k}^{\mathrm{NW}}-u_{j, k}^{\mathrm{NE}}+u_{j+1, k}^{\mathrm{SW}}-u_{j, k}^{\mathrm{SE}}\right],
\end{aligned}
$$

and

$$
\begin{aligned}
H_{j, k+1 / 2}^{y}:=\frac{b_{j, k+1 / 2}^{+}}{2\left(b_{j, k+1 / 2}^{+}-b_{j, k+1 / 2}^{-}\right)}\left[g\left(u_{j, k}^{\mathrm{NW}}\right)\right. & \left.+g\left(u_{j, k}^{\mathrm{NE}}\right)\right]-\frac{b_{j, k+1 / 2}^{-}}{2\left(b_{j, k+1 / 2}^{+}-b_{j, k+1 / 2}^{-}\right)}\left[g\left(u_{j, k+1}^{\mathrm{SW}}\right)+g\left(u_{j, k+1}^{\mathrm{SE}}\right)\right] \\
& +\frac{b_{j, k+1 / 2}^{+} b_{j, k+1 / 2}^{-}}{2\left(b_{j, k+1 / 2}^{+}-b_{j, k+1 / 2}^{-}\right)}\left[u_{j, k+1}^{\mathrm{SW}}-u_{j, k}^{\mathrm{NW}}+u_{j, k+1}^{\mathrm{SE}}-u_{j, k}^{\mathrm{NE}}\right] .
\end{aligned}
$$

Remark. The numerical fluxes in (2.12) and (2.13) offer a genuinely multidimensional discretization by adding the cross-diagonal directions to the Cartesian directions utilized in (2.8).

A Third-order Method. The third-order scheme is based on a reconstruction of a nonoscillatory piecewise quadratic polynomial. One of the possible ways to obtain an essentially nonoscillatory third-order reconstruction is by using a ENO or Weighted-ENO (WENO) approach presented in [26, 27]. For a general survey of the highly accurate ENO/WENO reconstructions we refer the reader to C.I.M.E. Lecture Notes [13, p. 333-381]. The disadvantage of the WENO-type interpolants in this context, however, is that they are based on smoothness indicators, and thus on an a priori information about the solution, which may be unavailable. This may result in spurious oscillations or extra smearing of discontinuities.

In this article, we have used an alternative reconstruction, which was proposed in [16]. The main idea is to apply 1-D nonoscillatory piecewise quadratic interpolants (for examples of such 1 -D reconstructions we refer the reader to $[28,11,16])$ in the $x$ - and $y$-directions, and in the diagonal directions. The detailed description of this 2-D extension can be found in [16]; see also [17]. 
The numerical fluxes, which correspond to the fourth-order Simpson's quadrature rule, are

$$
\begin{aligned}
H_{j+1 / 2, k}^{x}:= & \frac{a_{j+1 / 2, k}^{+}}{6\left(a_{j+1 / 2, k}^{+}-a_{j+1 / 2, k}^{-}\right)}\left[f\left(u_{j, k}^{\mathrm{NE}}\right)+4 f\left(u_{j, k}^{\mathrm{E}}\right)+f\left(u_{j, k}^{\mathrm{SE}}\right)\right] \\
& -\frac{a_{j+1 / 2, k}^{-}}{6\left(a_{j+1 / 2, k}^{+}-a_{j+1 / 2, k}^{-}\right)}\left[f\left(u_{j+1, k}^{\mathrm{NW}}\right)+4 f\left(u_{j+1, k}^{\mathrm{W}}\right)+f\left(u_{j+1, k}^{\mathrm{SW}}\right)\right] \\
& +\frac{a_{j+1 / 2, k}^{+} a_{j+1 / 2, k}^{-}}{6\left(a_{j+1 / 2, k}^{+}-a_{j+1 / 2, k}^{-}\right)}\left[u_{j+1, k}^{\mathrm{NW}}-u_{j, k}^{\mathrm{NE}}+4\left(u_{j+1, k}^{\mathrm{W}}-u_{j, k}^{\mathrm{E}}\right)+u_{j+1, k}^{\mathrm{SW}}-u_{j, k}^{\mathrm{SE}}\right],
\end{aligned}
$$

and

$$
\begin{aligned}
H_{j, i+1 / 2}^{y}:= & \frac{b_{j, k+1 / 2}^{+}}{6\left(b_{j, k+1 / 2}^{+}-b_{j, k+1 / 2}^{-}\right)}\left[g\left(u_{j, k}^{\mathrm{NW}}\right)+4 g\left(u_{j, k}^{\mathrm{N}}\right)+g\left(u_{j, k}^{\mathrm{NE}}\right)\right] \\
& -\frac{b_{j, k+1 / 2}^{-}}{6\left(b_{j, k+1 / 2}^{+}-b_{j, k+1 / 2}^{-}\right)}\left[g\left(u_{j, k+1}^{\mathrm{SW}}\right)+4 g\left(u_{j, k+1}^{\mathrm{S}}\right)+g\left(u_{j, k+1}^{\mathrm{SE}}\right)\right] \\
& +\frac{b_{j, k+1 / 2}^{+} b_{j, k+1 / 2}^{-}}{6\left(b_{j, k+1 / 2}^{+}-b_{j, k+1 / 2}^{-}\right)}\left[u_{j, k+1}^{\mathrm{SW}}-u_{j, k}^{\mathrm{NW}}+4\left(u_{j, k+1}^{\mathrm{S}}-u_{j, k}^{\mathrm{N}}\right)+u_{j, k+1}^{\mathrm{SE}}-u_{j, k}^{\mathrm{NE}}\right] .
\end{aligned}
$$

In (2.14)-(2.15), the one-sided local speeds $a_{j+1 / 2, k}^{ \pm}, b_{j, k+1 / 2}^{ \pm}$are defined in (2.8), and the values of the $u$ 's are computed in (2.7) and (2.11), using the piecewise quadratic reconstruction $\left\{p_{j, k}\right\}$ at time $t$.

\section{Remarks.}

1. Time integration. All the aforementioned schemes, (2.4), (2.9)-(2.10); (2.4), (2.12)(2.13), and (2.4), (2.14)-(2.15) are semi-discrete schemes. To solve the corresponding systems of time dependent ODEs, one may use any stable ODE solver. In the examples below, we use integrate the second- and third-order central schemes using, respectively, the second-order modified Euler time-discretization and the third-order TVD Runge-Kutta method [29, 30] (consult [13, pp. 384-394] for a general overview).

2. Simplicity. The Godunov-type central schemes enjoy the particular advantage that the computation of the midvalues in (2.7) and (2.11) is based on component-wise evaluation of the numerical derivatives (2.6). Consequently, no (approximate) Riemann problem solvers are required, and the intricate and time consuming part of computing the eigensystem of the problem at hand is avoided. In this sense, the simplicity offered by the above semi-discrete central schemes coupled with one's favorite ODEs solvers, leads to a class of easily implemented "black-box" methods for solving 1-D and 2-D systems of conservation laws and related equations governing the evolution of large gradient phenomena (see [14-17, 31]).

3. Upwinding. The schemes described above are so-called central schemes in the sense that their solution are realized in terms of cell averages which are integrated across the center of Riemann fans. At the same time, these schemes also share common features with the class of upwind schemes, most notably, their solution follow the propagation of left- and right-going waves emanating from the interfaces between interior discontinuities. These schemes are therefore referred to as central-upwind schemes in [17]. 
To illustrate our point, one may consider the scalar linear advection equation, $u_{t}+a u_{x}$ $+b u_{y}=0$ with, for example, positive constants $a$ and $b$. Then the first-order version of the central-upwind scheme becomes a standard first-order upwind scheme

$$
\frac{d}{d t} u_{j, k}(t)=-a \frac{u_{j, k}-u_{j-1, k}}{\Delta x}-b \frac{u_{j, k}-u_{j, k-1}}{\Delta y}
$$

4. Multidimensional approach. The second-order scheme (2.4), (2.9)-(2.10) can also be obtained using the so-called "dimension-by-dimension" approach, namely, by adding the corresponding 1-D central fluxes (similar to the derivation of multidimensional schemes in $[14,15])$.

The third-order scheme (2.4), (2.14)-(2.15), like the second-order scheme (2.4), (2.12)(2.13), however, are genuinely multidimensional because of the additional cross-diagonal terms; for details see [16, 17]. The performed numerical experiments indicate that the genuinely multidimensional second-order scheme (2.4), (2.12)-(2.13) is more stable and less sensitive to a choice of piecewise linear reconstruction than the dimension-bydimension scheme (2.4), (2.9)-(2.10).

5. Maximum principle. In the scalar case, both second-order schemes (2.4), (2.9)-(2.10) and (2.4), (2.12)-(2.13), coupled with the nonoscillatory minmod reconstruction (2.2)-(2.6), satisfy the maximum principle ([17, Theorem 3.1).

\section{NUMERICAL EXPERIMENTS}

Let us consider the 2-D Euler equations of gas dynamics,

$$
\begin{aligned}
\frac{\partial}{\partial t}\left[\begin{array}{c}
\rho \\
\rho u \\
\rho v \\
E
\end{array}\right]+\frac{\partial}{\partial x}\left[\begin{array}{c}
\rho u \\
\rho u^{2}+p \\
\rho u v \\
u(E+p)
\end{array}\right]+\frac{\partial}{\partial y}\left[\begin{array}{c}
\rho v \\
\rho u v \\
\rho v^{2}+p \\
v(E+p)
\end{array}\right] & =0, \\
p & =(\gamma-1) \cdot\left[E-\frac{\rho}{2}\left(u^{2}+v^{2}\right)\right],
\end{aligned}
$$

for an ideal gas, $\gamma=1.4$. Here $\rho, u, v, p$, and $E$ are the density, the $x$ - and $y$-velocities, the pressure and the total energy, respectively.

We solve the Riemann problem for (3.1) with initial data

$$
(p, \rho, u, v)(x, y, 0)= \begin{cases}\left(p_{1}, \rho_{1}, u_{1}, v_{1}\right), & \text { if } x>0.5 \text { and } y>0.5 \\ \left(p_{2}, \rho_{2}, u_{2}, v_{2}\right), & \text { if } x<0.5 \text { and } y>0.5 \\ \left(p_{3}, \rho_{3}, u_{3}, v_{3}\right), & \text { if } x<0.5 \text { and } y<0.5 \\ \left(p_{4}, \rho_{4}, u_{4}, v_{4}\right), & \text { if } x>0.5 \text { and } y<0.5\end{cases}
$$

According to [3, 5], there are 19 genuinely different admissible configurations for polytropic gas, separated by the three types of 1-D centered waves, namely, $\operatorname{rarefaction}-(\vec{R})$, shock- $(\bar{S})$, and contact-wave $\left(J^{ \pm}\right)$. The arrows $(\vec{\bullet})$ and $\left(^{*}\right)$ indicate forward and backward waves, and the superscript $J^{+}$and $J^{-}$refer to negative, respectively, positive contacts. Consult $[1,2,4,6]$ for details. 
In this section, we compute all these solutions using the second- and third-order genuinely multidimensional central schemes, (2.4), (2.12)-(2.13) and (2.4), (2.14)-(2.15). The computational configuration is identical to the one in [5]: the solution is computed using $400 \times 400$ gridpoints, and the results are recorded at the final time indicated as $\mathbf{T}$. The CFL number used is 0.475 . Our numerical examples below show the (uniformly distributed) density contour lines subject to 19 different initial data configurations, the same initial configurations as in [5], and we refer the reader to Schultz-Rinne et al. [2] for a detailed discussion on the wave formation in each of these configurations.

Below, we make brief comments for each configuration, comparing our computed results with the upwind computations in [2] and [5]. Overall, our results based on central schemes reveal the same detailed information on the variety of wave formations, in a complete agreement with the upwind schemes. It is rather remarkable that this amount of details is revealed without any input on the 1-D elementary waves involved, beyond the maximal local speeds. The high resolution in the central and upwind approaches is comparable, with the only noticeable difference in contacts and slip lines. As expected, the resolution of the corresponding linear waves by the upwind schemes, particularly in [2], is somewhat sharper than in the central computations. The difference in resolution of these linear waves is small and in fact, in certain cases, consult Configurations 8 and 17 below, the central schemes perform better than the results reported in [5].

\section{Configuration 1.}

$$
\vec{R}_{32} \underset{\vec{R}_{34}}{\overrightarrow{R_{21}}} \overrightarrow{R_{41}}
$$

The initial data are

$$
\begin{array}{llll}
p_{2}=0.4 & \rho_{2}=0.5197 & p_{1}=1 & \rho_{1}=1 \\
u_{2}=-0.7259 & v_{2}=0 & u_{1}=0 & v_{1}=0 \\
& & & \\
p_{3}=0.0439 & \rho_{3}=0.1072 & p_{4}=0.15 & \rho_{4}=0.2579 \\
u_{3}=-0.7259 & v_{3}=-1.4045 & u_{4}=0 & v_{4}=-1.4045
\end{array}
$$

Comments. We recover here the same "ripples" in the middle of the left and lower rarefactions observed in [5] and in a sharpened form in [2]. The computed front propagating in between these two rarefactions is in agreement with [5], and is sharper than the one reported in [2] [Fig. 3.1(a), 3.1(b)].

\section{Configuration 2.}

$$
\underset{R_{32}}{\stackrel{\leftarrow}{R_{34}}} \stackrel{\overrightarrow{R_{21}}}{R_{41}}
$$

The initial data are 

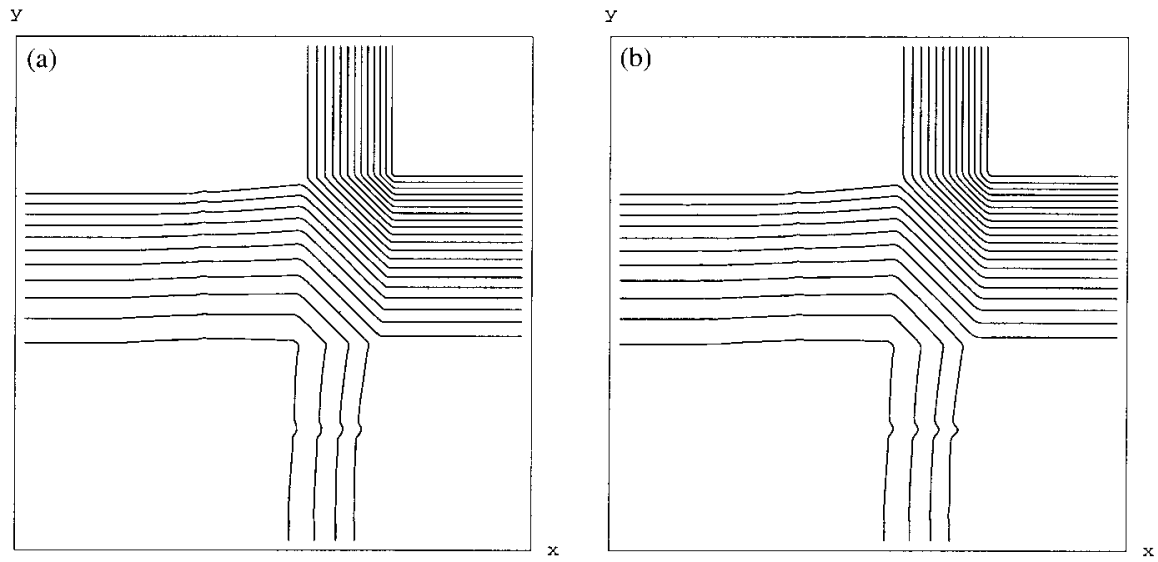

FIG. 3.1. (a) 2nd-order scheme, $\theta=2, \mathbf{T}=0.2$; (b) 3rd-order scheme, $\mathbf{T}=0.2$.

$$
\begin{array}{llll}
p_{2}=0.4 & \rho_{2}=0.5197 & p_{1}=1 & \rho_{1}=1 \\
u_{2}=-0.7259 & v_{2}=0 & u_{1}=0 & v_{1}=0 \\
& & & \\
p_{3}=1 & \rho_{3}=1 & p_{4}=0.4 & \rho_{4}=0.5197 \\
u_{3}=-0.7259 & v_{3}=-0.7259 & u_{4}=0 & v_{4}=-0.7259
\end{array}
$$

Comments. The $\theta$-limiter (2.6) proves to be over-compressive with $\theta=2$; the spurious oscillations can be noticed on the left [Fig. 3.2(a)] are avoided in the third-order computation on the right [Fig. 3.2(b)]. The same secondary "ripples" are observed in all the computations.

\section{Configuration 3.}

$$
\underset{S_{32}}{\overleftarrow{S_{34}}} \stackrel{\overleftarrow{S_{21}}}{\overleftarrow{S_{41}}}
$$

y

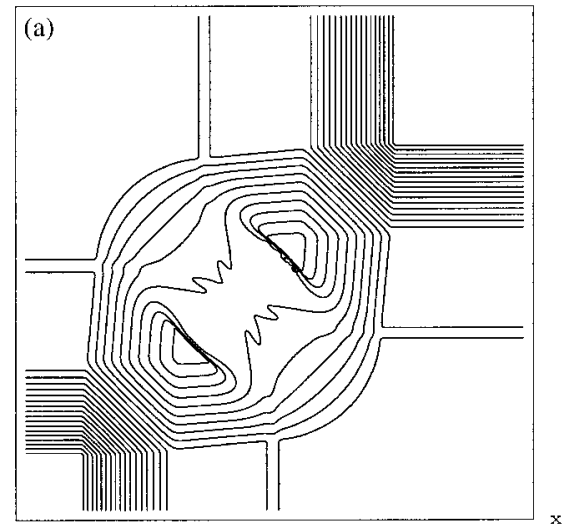

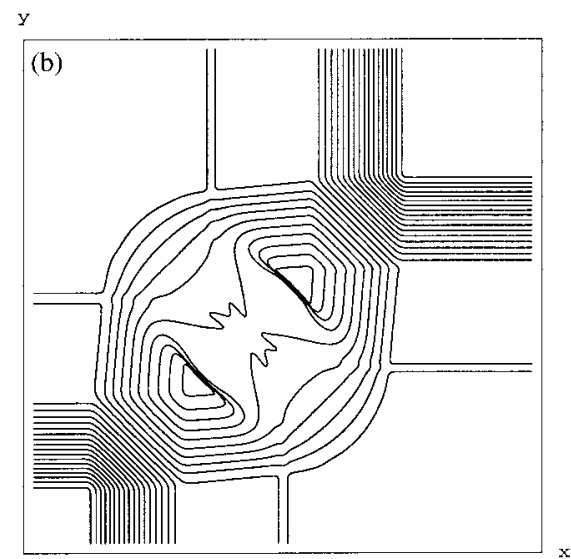

FIG. 3.2. (a) 2nd-order scheme, $\theta=2, \mathbf{T}=0.2$; (b) 3rd-order scheme, $\mathbf{T}=0.2$. 

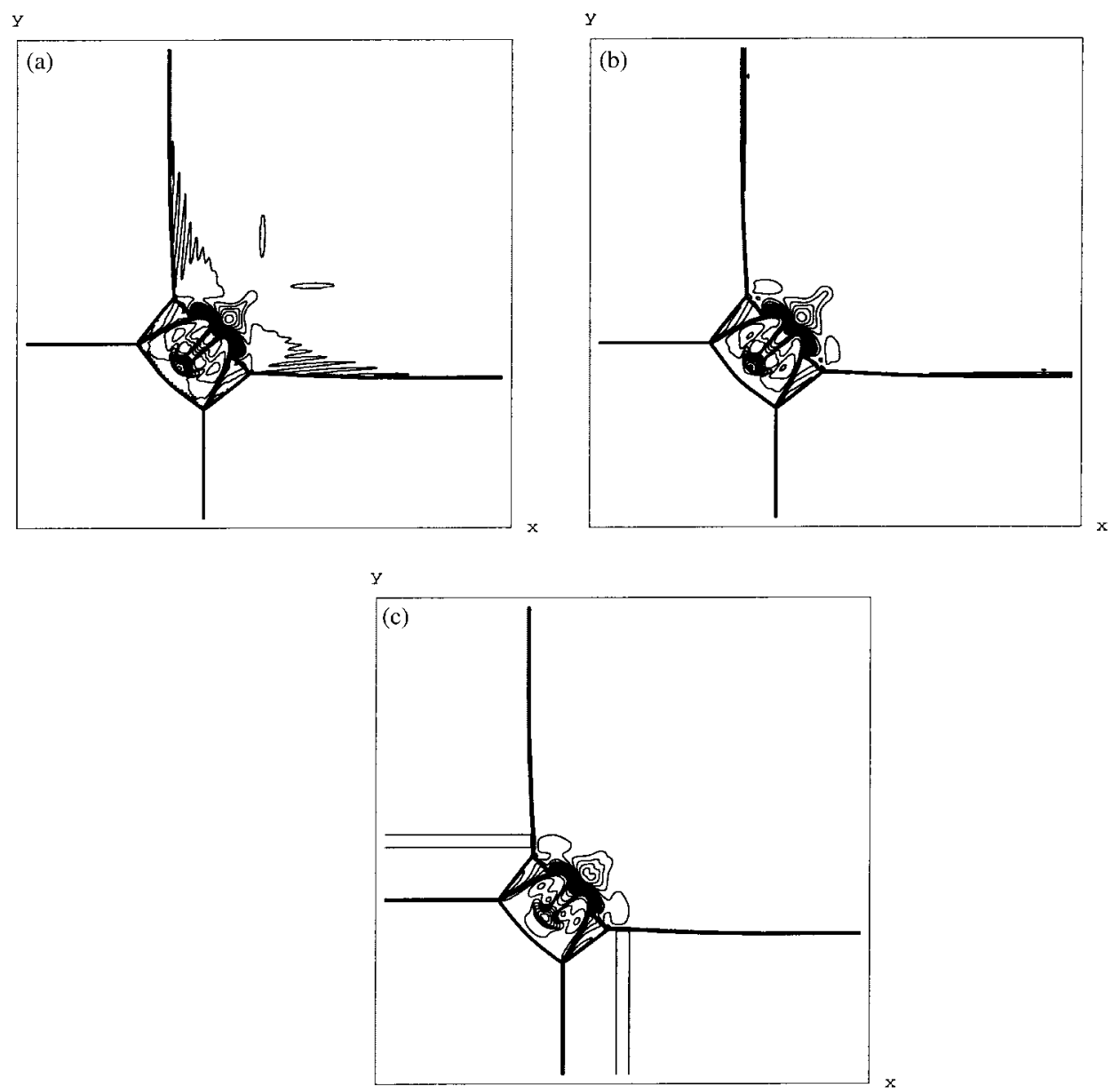

FIG. 3.3. (a) 2nd-order scheme, $\theta=2, \mathbf{T}=0.3$; (b) 3rd-order scheme, $\mathbf{T}=0.3$; (c) 2nd-order scheme, $\theta=$ $1, \mathbf{T}=0.3$.

The initial data are

$$
\begin{array}{llll}
p_{2}=0.3 & \rho_{2}=0.5323 & p_{1}=1.5 & \rho_{1}=1.5 \\
u_{2}=1.206 & v_{2}=0 & u_{1}=0 & v_{1}=0 \\
& & & \\
p_{3}=0.029 & \rho_{3}=0.138 & p_{4}=0.3 & \rho_{4}=0.5323 \\
u_{3}=1.206 & v_{3}=1.206 & u_{4}=0 & v_{4}=1.206
\end{array}
$$

Comments. As before, oscillations because of the over-compressive limiter with $\theta=2$ in Figure 3.3(a) are reduced in the third-order case, and even sharper results are obtained with a more "mild" limiter parameter, $\theta=1$. The resolution of shocks is comparable to the upwind results. 

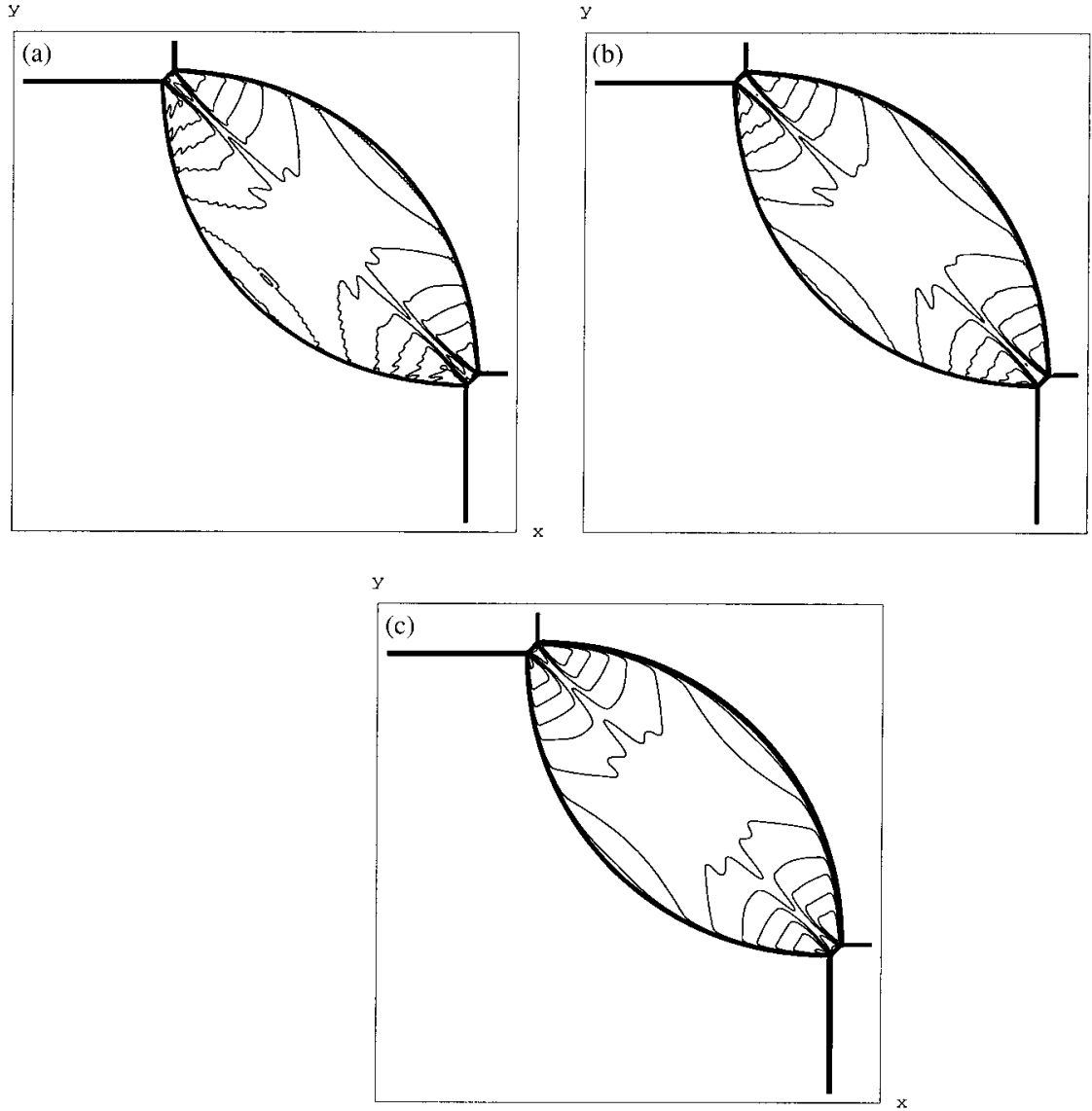

FIG. 3.4. (a) 2nd-order scheme, $\theta=2, \mathbf{T}=0.25$; (b) 3rd-order scheme, $\mathbf{T}=0.25$; (c) 2nd-order scheme, $\theta=1, \mathbf{T}=0.25$.

\section{Configuration 4.}

$$
\overrightarrow{S_{32}} \underset{S_{34}}{\overleftarrow{S_{21}}} \stackrel{\overleftarrow{S_{41}}}{\overleftarrow{\leftarrow}}
$$

The initial data are

$$
\begin{array}{llll}
p_{2}=0.35 & \rho_{2}=0.5065 & p_{1}=1.1 & \rho_{1}=1.1 \\
u_{2}=0.8939 & v_{2}=0 & u_{1}=0 & v_{1}=0 \\
& & & \\
p_{3}=1.1 & \rho_{3}=1.1 & p_{4}=0.35 & \rho_{4}=0.5065 \\
u_{3}=0.8939 & v_{3}=0.8939 & u_{4}=0 & v_{4}=0.8939
\end{array}
$$



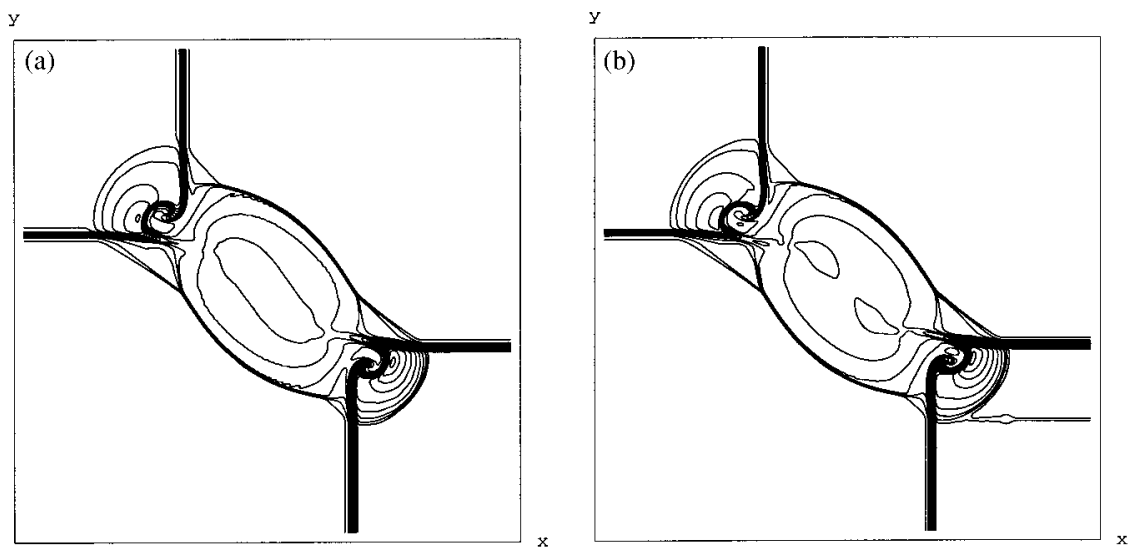

FIG. 3.5. (a) 2nd-order scheme, $\theta=1.3, \mathbf{T}=0.23$; (b) 3rd-order scheme, $\mathbf{T}=0.23$.

Comments. Again, $\theta=2$ is over-compressive in Figure 3.4(a), the oscillations are reduced in the third-order approximation, and sharp results, in complete agreement with those of $[2,5]$, are obtained with the usual minmod limiter, corresponding to $\theta=1$.

\section{Configuration 5.}

$$
\begin{array}{lll}
J_{32}^{-} & J_{21}^{-} & \\
& J_{34}^{-} & J_{41}^{-}
\end{array}
$$

The initial data are

$$
\begin{array}{llll}
p_{2}=1 & \rho_{2}=2 & p_{1}=1 & \rho_{1}=1 \\
u_{2}=-0.75 & v_{2}=0.5 & u_{1}=-0.75 & v_{1}=-0.5 \\
p_{3}=1 & \rho_{3}=1 & p_{4}=1 & \rho_{4}=3 \\
u_{3}=0.75 & v_{3}=0.5 & u_{4}=0.75 & v_{4}=-0.5
\end{array}
$$

Comments. Same features are picked up by al methods, with similar resolution as in [5]. The contact obtained in [2] has a better resolution [Fig. 3.5(a,b)].

\section{Configuration 6.}

$$
\begin{array}{ccc}
J_{32}^{+} & J_{21}^{-} & \\
& J_{34}^{-} & J_{41}^{+}
\end{array}
$$

The initial data are

$$
\begin{array}{llll}
p_{2}=1 & \rho_{2}=2 & p_{1}=1 & \rho_{1}=1 \\
u_{2}=0.75 & v_{2}=0.5 & u_{1}=0.75 & v_{1}=-0.5 \\
p_{3}=1 & \rho_{3}=1 & p_{4}=1 & \rho_{4}=3 \\
u_{3}=-0.75 & v_{3}=0.5 & u_{4}=-0.75 & v_{4}=-0.5
\end{array}
$$



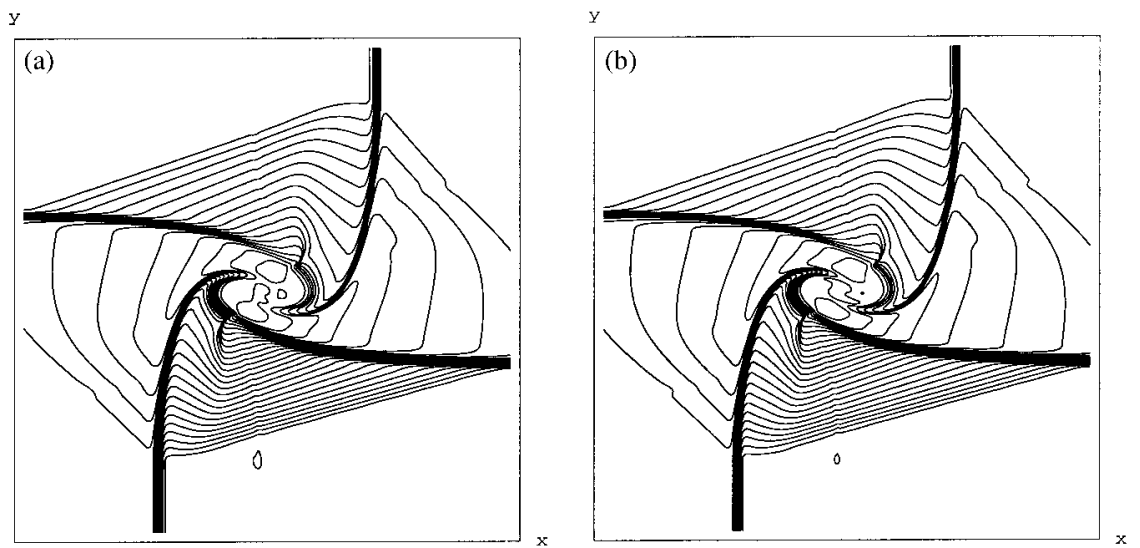

FIG. 3.6. (a) 2nd-order scheme, $\theta=1.3 ; \mathbf{T}=0.3$; (b) 3rd-order scheme, $\mathbf{T}=0.3$.

Comments. The ripples observed in both the NE and SW quadrants, are recovered with a comparable resolution to the one in $[2,5]$ [Fig. 3.6(a,b)].

\section{Configuration 7.}

$$
\begin{array}{ccc}
J_{32}^{-} & \overrightarrow{R_{21}} & \\
& & \overrightarrow{R_{41}} \\
& J_{34}^{-} &
\end{array}
$$

The initial data are

$$
\begin{array}{llll}
p_{2}=0.4 & \rho_{2}=0.5197 & p_{1}=1 & \rho_{1}=1 \\
u_{2}=-0.6259 & v_{2}=0.1 & u_{1}=0.1 & v_{1}=0.1 \\
& & & \\
p_{3}=0.4 & \rho_{3}=0.8 & p_{4}=0.4 & \rho_{4}=0.5197 \\
u_{3}=0.1 & v_{3}=0.1 & u_{4}=0.1 & v_{4}=-0.6259
\end{array}
$$

Comments. The high-resolution is in agreement with the corresponding upwind results in [5]. The contacts in [2] are sharper [Fig. 3.7(a,b)].

\section{Configuration 8.}

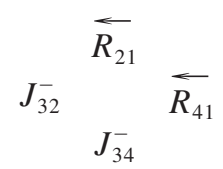

The initial data are

$$
\begin{array}{llll}
p_{2}=1 & \rho_{2}=1 & p_{1}=0.4 & \rho_{1}=0.5197 \\
u_{2}=-0.6259 & v_{2}=0.1 & u_{1}=0.1 & v_{1}=0.1 \\
& & & \\
p_{3}=1 & \rho_{3}=0.8 & p_{4}=1 & \rho_{4}=1 \\
u_{3}=0.1 & v_{3}=0.1 & u_{4}=0.1 & v_{4}=-0.6259
\end{array}
$$



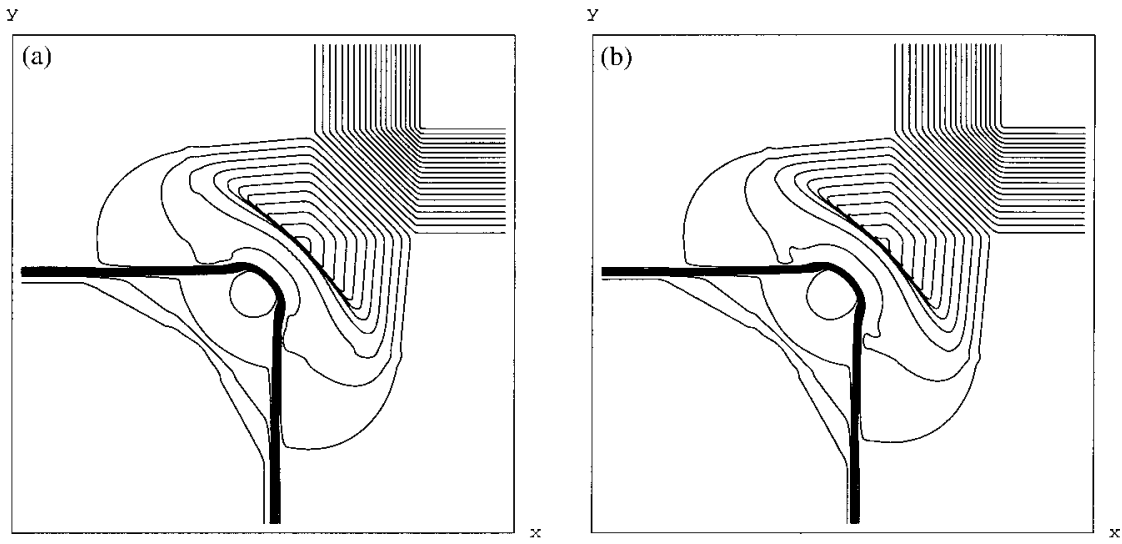

FIG. 3.7. (a) 2nd-order scheme, $\theta=1.3, \mathbf{T}=0.25$; (b) 3rd-order scheme, $\mathbf{T}=0.25$.

Comments. The semi-circular wavefront is recovered here with sharper resolution than the one in [5], mainly due to the "genuinely multidimensional" approach taken here, in terms of the cross-diagonal differences. Again, the bottom and left contacts are sharper in [2] [Fig. 3.8(a,b)].

\section{Configuration 9.}

$$
\begin{array}{lll}
\vec{R}_{32} & J_{21}^{+} & \\
& J_{34}^{+} &
\end{array}
$$

The initial data are

$$
\begin{array}{llll}
p_{2}=1 & \rho_{2}=2 & p_{1}=1 & \rho_{1}=1 \\
u_{2}=0 & v_{2}=-0.3 & u_{1}=0 & v_{1}=0.3 \\
p_{3}=0.4 & \rho_{3}=1.039 & p_{4}=0.4 & \rho_{4}=0.5197 \\
u_{3}=0 & v_{3}=-0.8133 & u_{4}=0 & v_{4}=-0.4259
\end{array}
$$
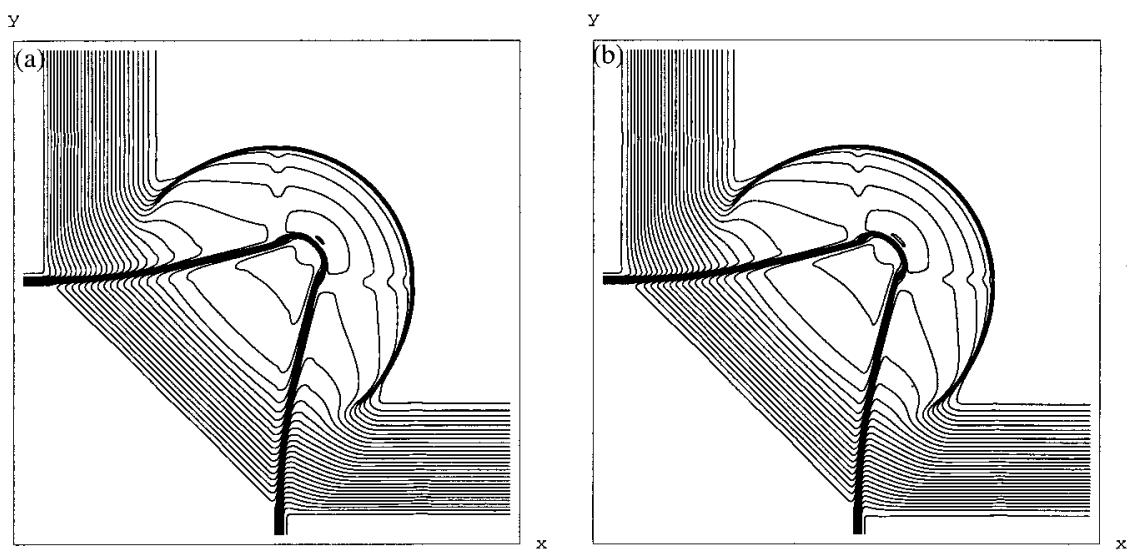

FIG. 3.8. (a) 2nd-order scheme, $\theta=1.3, \mathbf{T}=0.25$; (b) 3rd-order scheme, $\mathbf{T}=0.25$. 

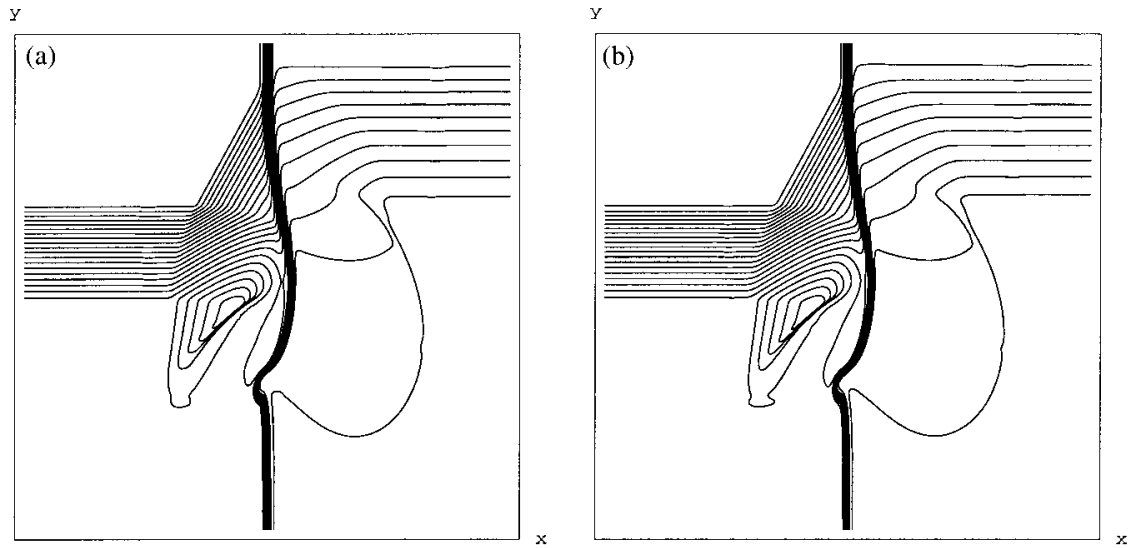

FIG. 3.9. (a) 2nd-order scheme, $\theta=1.3, \mathbf{T}=0.3$; (b) 3rd-order scheme, $\mathbf{T}=0.3$.

Comments. As typical with the upwind approach, contacts are resolved better in $[29,5]$. The "bulge" on the SW corner is identical in both central and upwind computations [Fig. 3.9(a,b)].

\section{Configuration 10.}

$$
\begin{array}{lll}
\vec{R}_{32} & J_{21}^{-} & \\
& J_{34}^{+} &
\end{array}
$$

The initial data are

$$
\begin{array}{llll}
p_{2}=1 & \rho_{2}=0.5 & p_{1}=1 & \rho_{1}=1 \\
u_{2}=0 & v_{2}=0.6076 & u_{1}=0 & v_{1}=0.4297 \\
& & & \\
p_{3}=0.3333 & \rho_{3}=0.2281 & p_{4}=0.3333 & \rho_{4}=0.4562 \\
u_{3}=0 & v_{3}=-0.6076 & u_{4}=0 & v_{4}=-0.4297
\end{array}
$$

Comments. There is a sharp resolution of the contact waves, but the resolution in [5] is somewhat better [Fig. 3.10(a,b)].

\section{Configuration 11.}

$$
J_{32}^{+} \underset{J_{34}^{+}}{\stackrel{S_{21}}{S_{S_{41}}}} \stackrel{ }{\leftarrow}
$$

The initial data are 

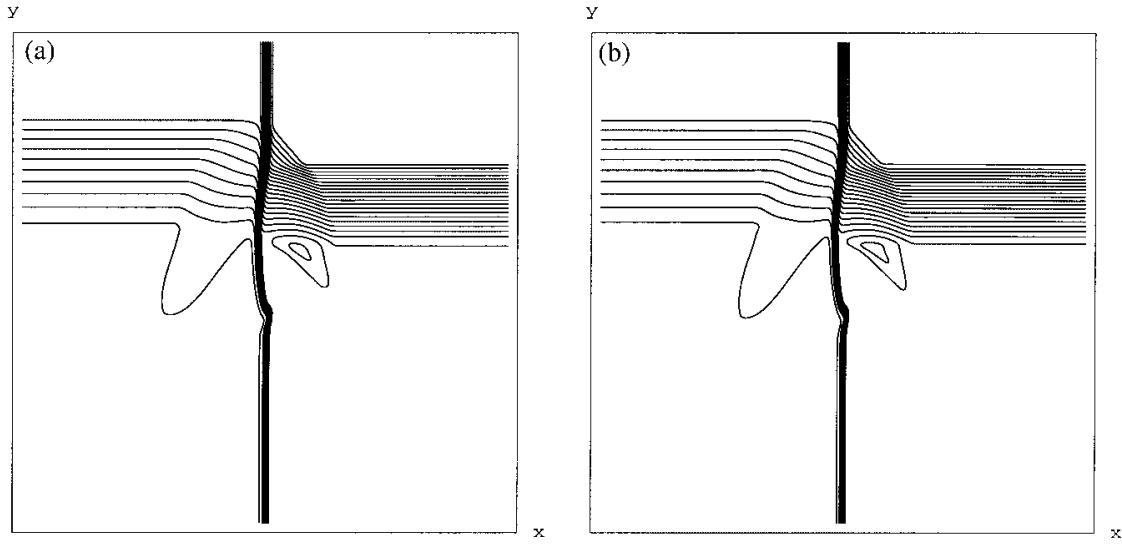

FIG. 3.10. (a) 2nd-order scheme, $\theta=1.3$, $\mathbf{T}=0.15$; (b) 3rd-order scheme, $\mathbf{T}=0.15$.

$$
\begin{array}{llll}
p_{2}=0.4 & \rho_{2}=0.5313 & p_{1}=1 & \rho_{1}=1 \\
u_{2}=0.8276 & v_{2}=0 & u_{1}=0.1 & v_{1}=0 \\
& & & \\
p_{3}=0.4 & \rho_{3}=0.8 & p_{4}=0.4 & \rho_{4}=0.5313 \\
u_{3}=0.1 & v_{3}=0 & u_{4}=0.1 & v_{4}=0.7276
\end{array}
$$

Comments. The "ripples" in the NE quadrant are captured in full agreement with [5]. The same results are strongly peaked in [2]. The limiter parameter $\theta=1.3$ as well as the third-order results lead to oscillations that are avoided with the standard minmod $(\theta=1)$ limiter. The contact on the left, however, is further smeared compared with [2, 5] [Fig. 3.11(a-c)].

\section{Configuration 12.}

$$
\begin{array}{ccc} 
& \overrightarrow{S_{21}} & \\
J_{32}^{+} & & \overrightarrow{S_{41}} \\
& J_{34}^{+} &
\end{array}
$$

The initial data are

$$
\begin{array}{llll}
p_{2}=1 & \rho_{2}=1 & p_{1}=0.4 & \rho_{1}=0.5313 \\
u_{2}=0.7276 & v_{2}=0 & u_{1}=0 & v_{1}=0 \\
p_{3}=1 & \rho_{3}=0.8 & p_{4}=1 & \rho_{4}=1 \\
u_{3}=0 & v_{3}=0 & u_{4}=0 & v_{4}=0.7276
\end{array}
$$

Comments. The resolution of the two contacts is improved by the third-order scheme, compared to the second-order one. The results are in agreement with upwind computations [Fig. $3.12(a, b)]$. 

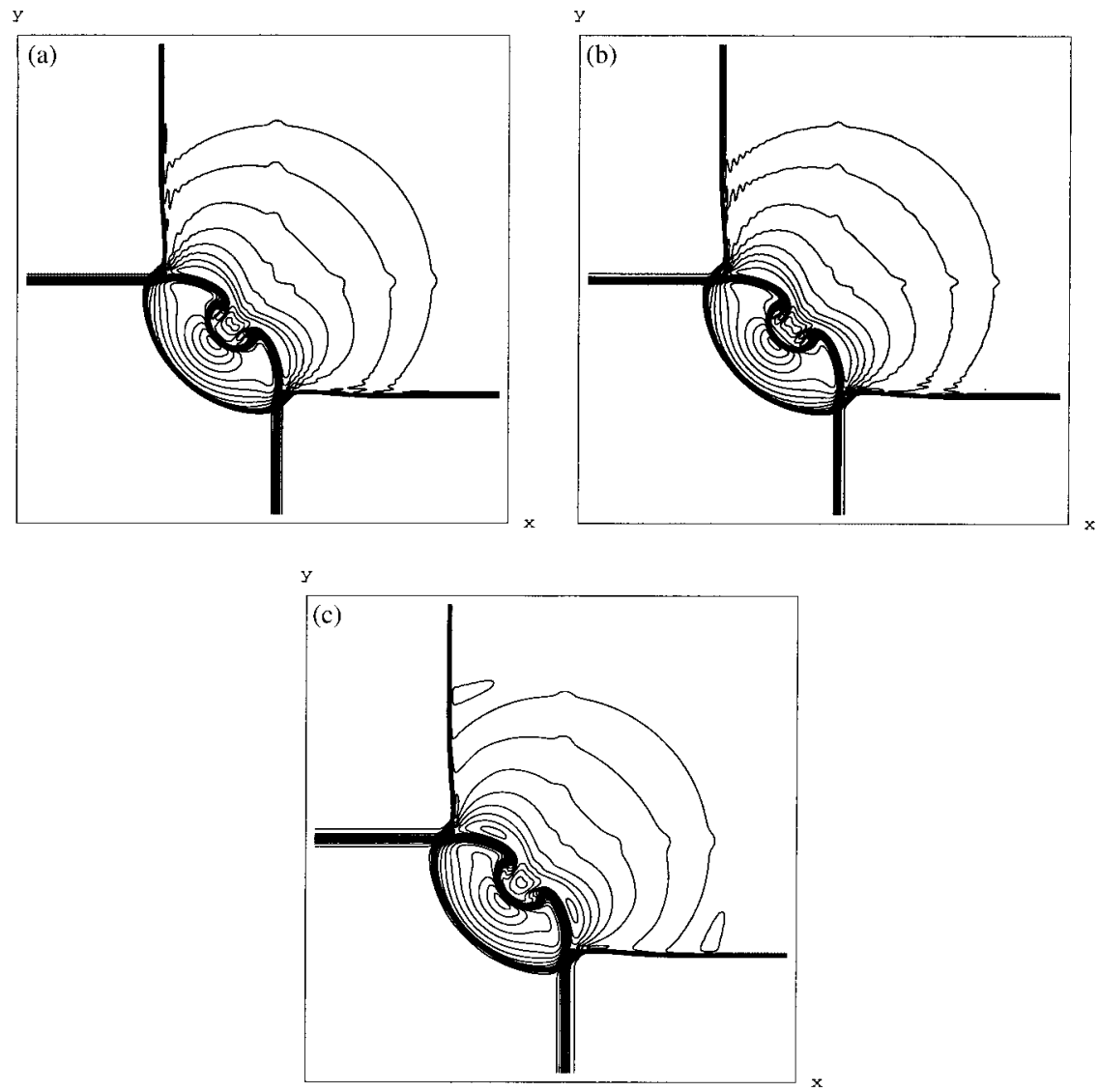

FIG. 3.11. (a) 2nd-order scheme, $\theta=1.3, \mathbf{T}=0.3$; (b) 3rd-order scheme, $\mathbf{T}=0.3$; (c) 2nd-order scheme, $\% \mathrm{v}=1, \mathbf{T}=0.3$.

\section{Configuration 13.}

$$
\overleftarrow{S}_{32} J_{J_{34}^{-}}^{J_{21}^{-}} \overleftarrow{S_{41}}
$$

The initial data are

$$
\begin{array}{llll}
p_{2}=1 & \rho_{2}=2 & p_{1}=1 & \rho_{1}=1 \\
u_{2}=0 & v_{2}=0.3 & u_{1}=0 & v_{1}=-0.3 \\
p_{3}=0.4 & \rho_{3}=1.0625 & p_{4}=0.4 & \rho_{4}=0.5313 \\
u_{3}=0 & v_{3}=0.8145 & u_{4}=0 & v_{4}=0.4276
\end{array}
$$

Comments. Should the blip in the NE quadrant should be there? Indeed, this is in agreement with [2] and [5] [Fig. 3.13(a,b)]. 

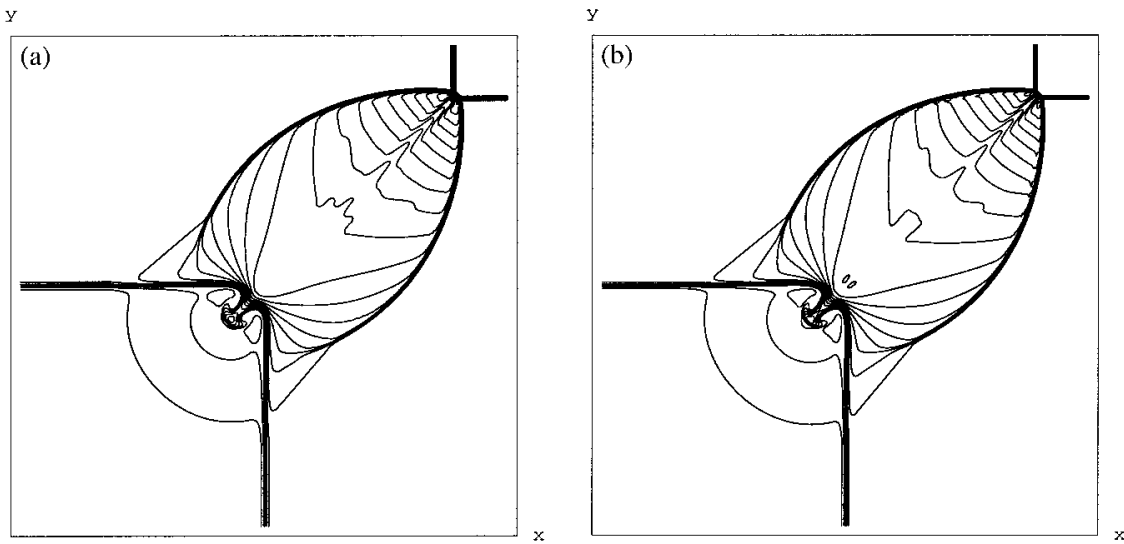

FIG. 3.12. (a) 2nd-order scheme, $\theta=1.3, \mathbf{T}=0.25$; (b) 3rd-order scheme, $\mathbf{T}=0.25$.

\section{Configuration 14.}

$$
\underset{S_{32}}{\leftarrow} \underset{J_{34}^{-}}{J_{21}^{+}} \stackrel{ }{S_{41}}
$$

The initial data are

$$
\begin{array}{llll}
p_{2}=8 & \rho_{2}=1 & p_{1}=8 & \rho_{1}=2 \\
u_{2}=0 & v_{2}=-1.2172 & u_{1}=0 & v_{1}=-0.5606 \\
& & & \\
p_{3}=2.6667 & \rho_{3}=0.4736 & p_{4}=2.6667 & \rho_{4}=0.9474 \\
u_{3}=0 & v_{3}=1.2172 & u_{4}=0 & v_{4}=1.1606
\end{array}
$$
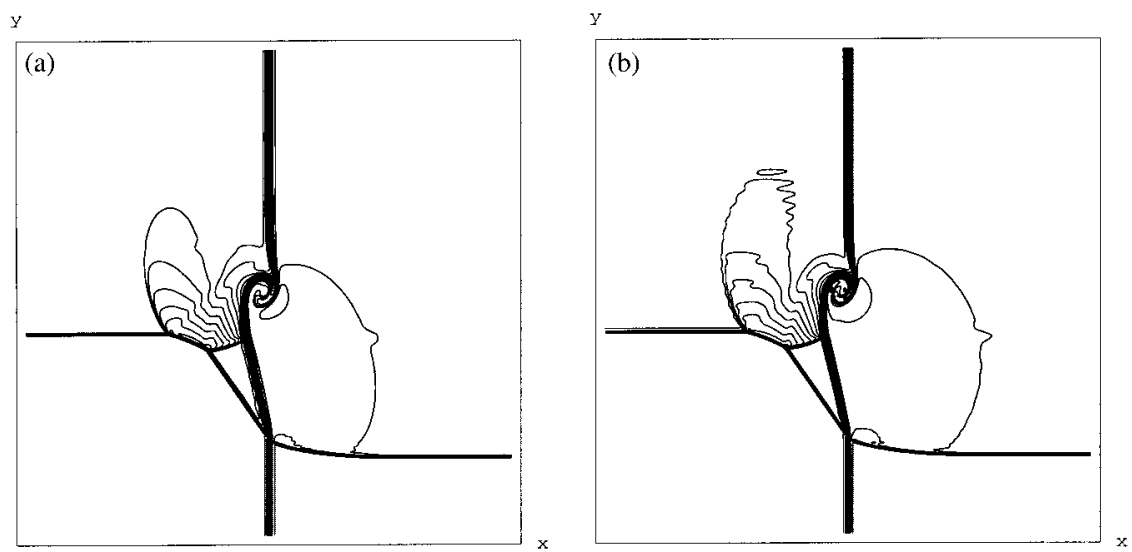

FIG. 3.13. (a) 2nd-order scheme, $\theta=1.3, \mathbf{T}=0.3$; (b) 3rd-order scheme, $\mathbf{T}=0.3$. 

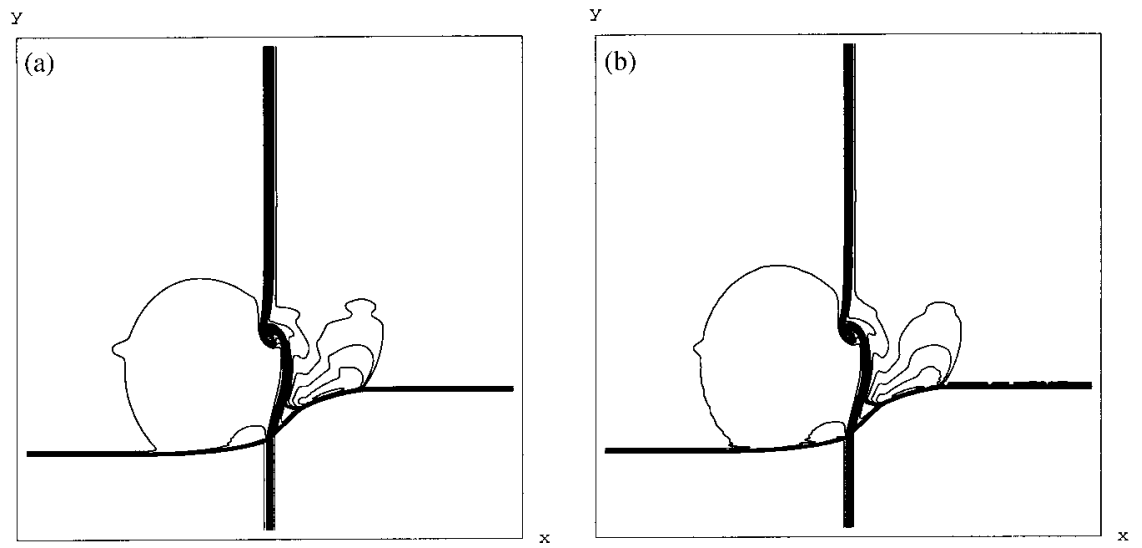

FIG. 3.14. (a) 2nd-order scheme, $\theta=1.3, \mathbf{T}=0.1$; (b) 3rd-order scheme, $\mathbf{T}=0.1$.

Comments. The resolution of the contact in [5] is slightly sharper than the one achieved by the central scheme [Fig. 3.14(a,b)].

\section{Configuration 15.}

$$
\begin{array}{ccc} 
& \overrightarrow{R_{21}} & \\
J_{32}^{-} & & \overleftarrow{S_{41}} \\
& J_{34}^{+} &
\end{array}
$$

The initial data are

$$
\begin{array}{llll}
p_{2}=0.4 & \rho_{2}=0.5197 & p_{1}=1 & \rho_{1}=1 \\
u_{2}=-0.6259 & v_{2}=-0.3 & u_{1}=0.1 & v_{1}=-0.3 \\
& & & \\
p_{3}=0.4 & \rho_{3}=0.8 & p_{4}=0.4 & \rho_{4}=0.5313 \\
u_{3}=0.1 & v_{3}=-0.3 & u_{4}=0.1 & v_{4}=0.4276
\end{array}
$$

Comments. Again, the sharp resolution of the contacts is only slightly less than those in [5]. The lower contact in [2] is sharper, but our result is free of the weak oscillations observed in [2] at the tip of the shock [Fig. 3.15(a,b)].

\section{Configuration 16.}

$$
\begin{array}{ccc}
J_{32}^{-} & \overleftarrow{R_{21}} & \\
& & J_{34}^{+}
\end{array}
$$

The initial data are 

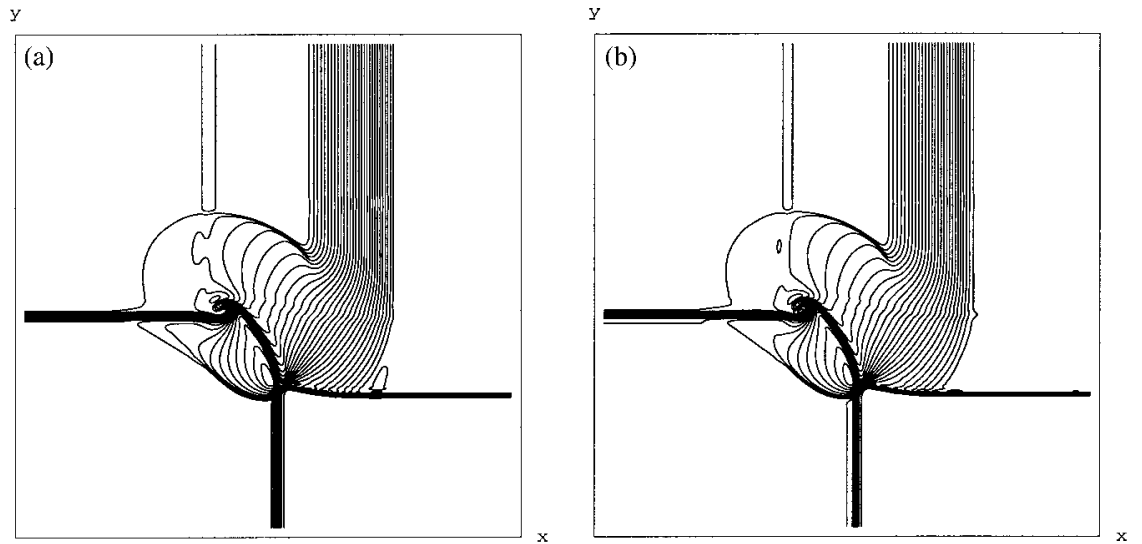

FIG. 3.15. (a) 2nd-order scheme, $\theta=1.3, \mathbf{T}=0.2$; (b) 3rd-order scheme, $\mathbf{T}=0.2$.

$$
\begin{array}{llll}
p_{2}=1 & \rho_{2}=1.0222 & p_{1}=0.4 & \rho_{1}=0.5313 \\
u_{2}=-0.6179 & v_{2}=0.1 & u_{1}=0.1 & v_{1}=0.1 \\
& & & \\
p_{3}=1 & \rho_{3}=0.8 & p_{4}=1 & \rho_{4}=1 \\
u_{3}=0.1 & v_{3}=0.1 & u_{4}=0.1 & v_{4}=0.8276
\end{array}
$$

Comments. The ripples, observed between the shock and contact waves, reproduce the same waveform as in [2, 5]. Here, the shock resolution in [2] is sharper than [5] and the result in Figure 3.16(b).

\section{Configuration 17.}

$$
\underset{S_{32}}{\leftarrow} \underset{J_{34}^{-}}{J_{21}^{-}} \overrightarrow{R_{41}}
$$
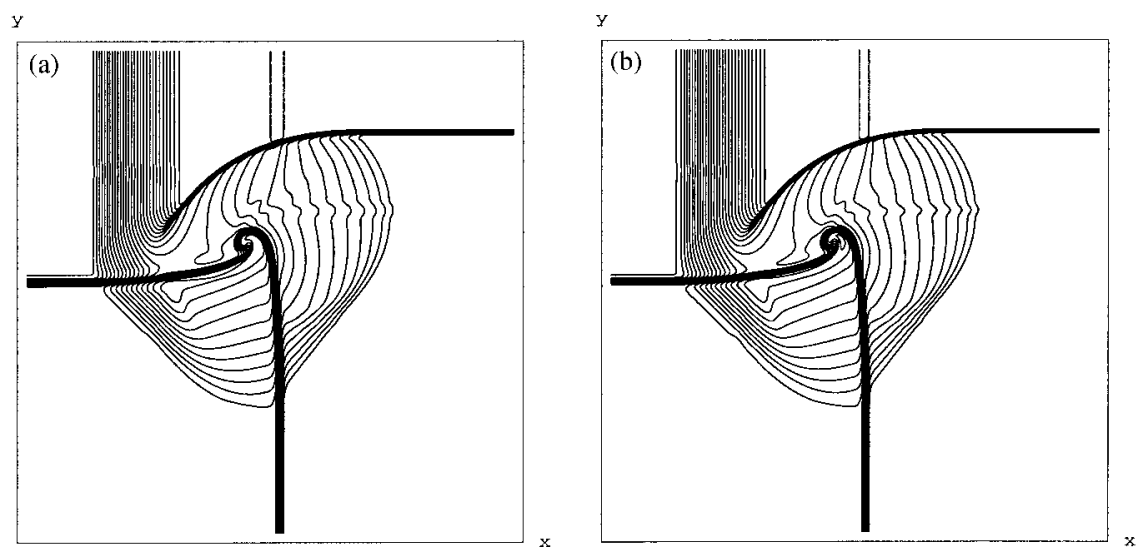

FIG. 3.16. (a) 2nd-order scheme, $\theta=1.3, \mathbf{T}=0.2$; (b) 3rd-order scheme, $\mathbf{T}=0.2$. 

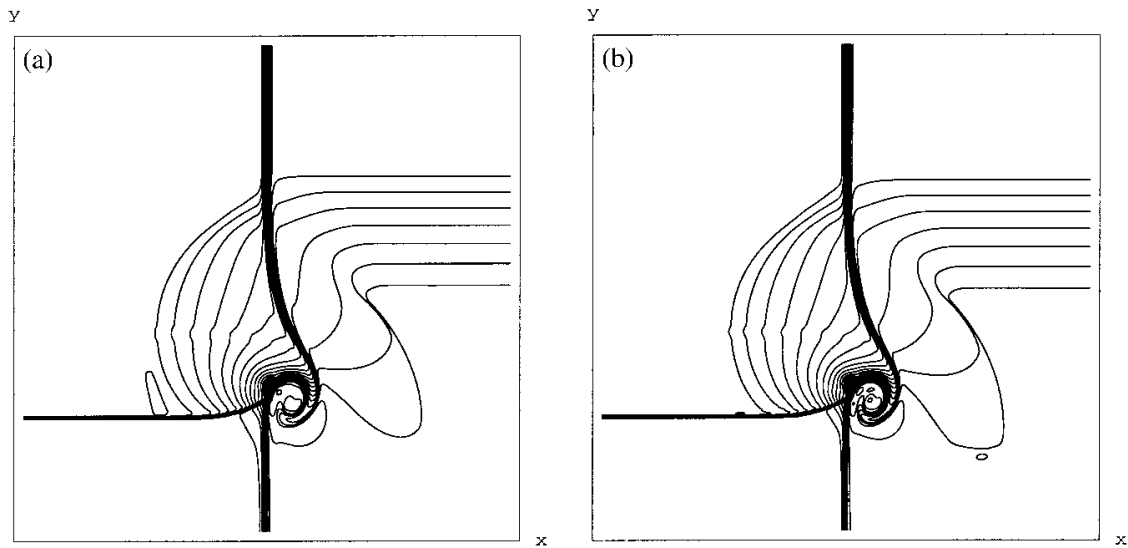

FIG. 3.17. (a) 2nd-order scheme, $\theta=1.3, \mathbf{T}=0.3$; (b) 3rd-order scheme, $\mathbf{T}=0.3$.

The initial data are

$$
\begin{array}{llll}
p_{2}=1 & \rho_{2}=2 & p_{1}=1 & \rho_{1}=1 \\
u_{2}=0 & v_{2}=-0.3 & u_{1}=0 & v_{1}=-0.4 \\
p_{3}=0.4 & \rho_{3}=1.0625 & p_{4}=0.4 & \rho_{4}=0.5197 \\
u_{3}=0 & v_{3}=0.2145 & u_{4}=0 & v_{4}=-1.1259
\end{array}
$$

Comments. Here, we obtain sharp resolution of the contact without the spurious vorticities appearing in [5]. In both cases, one observes the ripple formed in the NW quadrant [Fig. $3.17(a, b)]$.

\section{Configuration 18.}

$$
\underset{S_{32}}{J_{34}^{+}} \stackrel{J_{21}^{+}}{\overrightarrow{R_{41}}}
$$

The initial data are

$$
\begin{array}{llll}
p_{2}=1 & \rho_{2}=2 & p_{1}=1 & \rho_{1}=1 \\
u_{2}=0 & v_{2}=-0.3 & u_{1}=0 & v_{1}=1 \\
p_{3}=0.4 & \rho_{3}=1.0625 & p_{4}=0.4 & \rho_{4}=0.5197 \\
u_{3}=0 & v_{3}=0.2145 & u_{4}=0 & v_{4}=0.2741
\end{array}
$$

Comments. The resolution of the contacts is almost as sharp as in [5]. The ripples in the NW quadrant are observed in all computations [Fig. 3.18(a,b)]. 

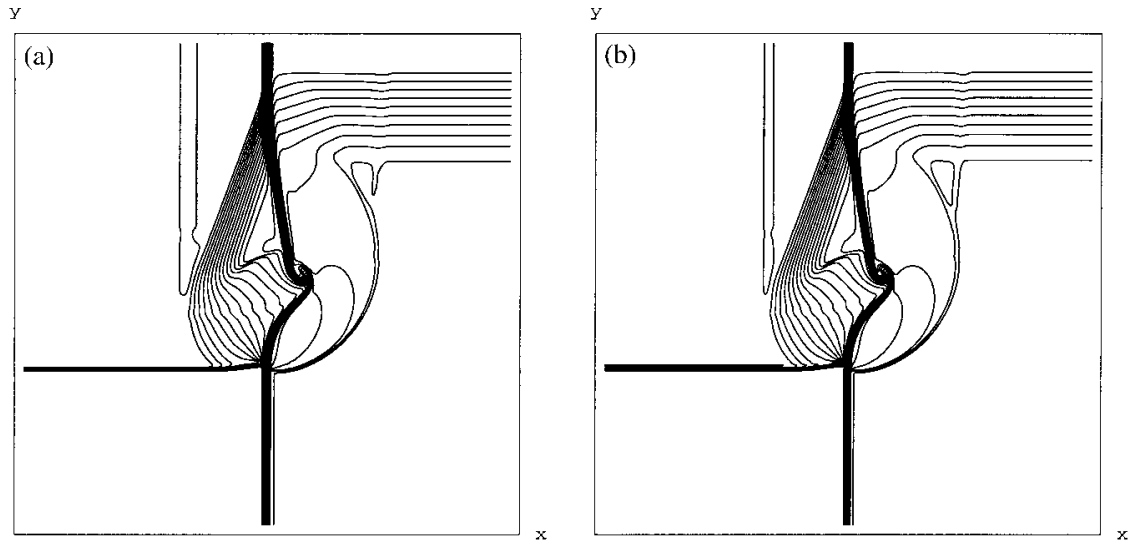

FIG. 3.18. (a) 2nd-order scheme, $\theta=1.3, \mathbf{T}=0.2$; (b) 3rd-order scheme, $\mathbf{T}=0.2$.

\section{Configuration 19.}

$$
\underset{S_{32}}{\leftarrow} \underset{J_{34}^{-}}{J_{21}^{+}} \overrightarrow{R_{41}}
$$

The initial data are

$$
\begin{array}{llll}
p_{2}=1 & \rho_{2}=2 & p_{1}=1 & \rho_{1}=1 \\
u_{2}=0 & v_{2}=-0.3 & u_{1}=0 & v_{1}=0.3 \\
p_{3}=0.4 & \rho_{3}=1.0625 & p_{4}=0.4 & \rho_{4}=0.5197 \\
u_{3}=0 & v_{3}=0.2145 & u_{4}=0 & v_{4}=-0.4259
\end{array}
$$
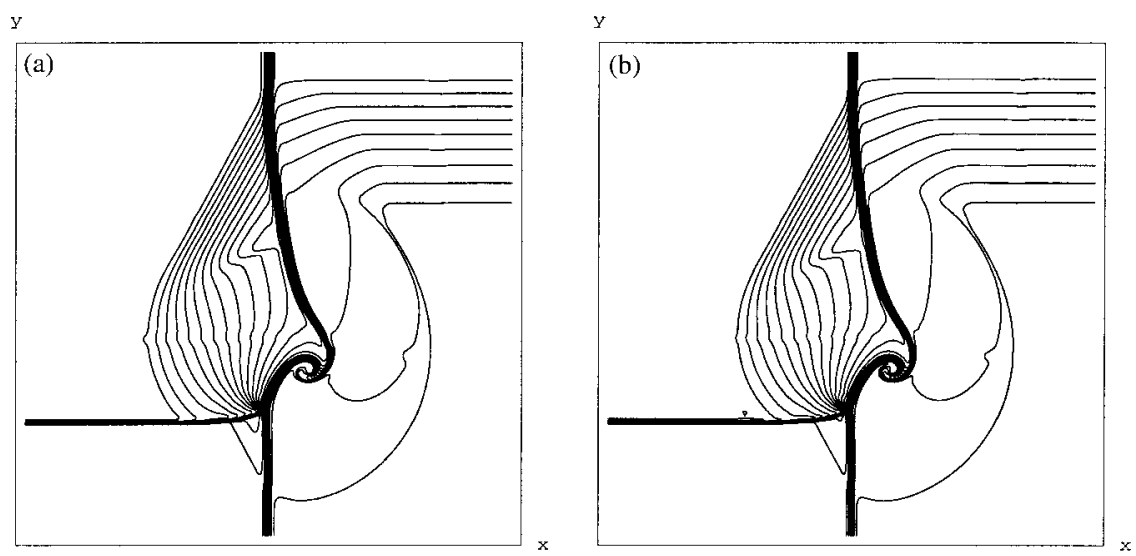

FIG. 3.19. (a) 2nd-order scheme, $\theta=1.3, \mathbf{T}=0.3$; (b) 3rd-order scheme, $\mathbf{T}=0.3$. 
Comments. As before, ripples are observed in NW quadrant, and only the resolution of contacts is slightly sharper in [5] [Fig. 3.19(a,b)].

\section{References}

1. C. W. Schulz-Rinne, Classification of the Riemann problem for two-dimensional gas dynamics, SIAM J Math Anal 24 (1993), 76-88.

2. C. W. Schulz-Rinne, J. P. Collins, and H. M. Glaz, Numerical solution of the Riemann problem for two-dimensional gas dynamics, SIAM J Sci Comp 14 (1993), 1394-1414.

3. T. Chang, G.-Q. Chen, and S. Yang, On the 2-D Riemann problem for the compressible Euler equations. I. Interaction of shocks and rarefaction waves, Discrete Contin Dynam Systems 1 (1995), $555-584$.

4. T. Zhang and Y. Zheng, Conjecture on the structure of solutions of the Riemann problem for two-dimensional gas dynamics systems, SIAM J Math Anal 21 (1990), 593-630.

5. P. Lax and X.-D. Liu, Solution of two-dimensional Riemann problems of gas dynamics by positive schemes, SIAM J Sci Comp 19 (1998), 319-340.

6. T. Chang, G.-Q. Chen, and S. Yang, On the 2-D Riemann problem for the compressible Euler equations. I. Interaction of contact discontinuities, Discrete Contin Dynam Systems 6 (2000), 419430.

7. P. D. Lax, Weak solutions of non-nonlinear hyperbolic equations and their numerical computations, Comm Pure Appl Math 7 (1954), 159-193.

8. S. K. Godunov, A finite difference method for the numerical computation of discontinuous solutions of the equations of fluid dynamics, Mat Sb 47 (1959), 271-290.

9. J. Smoller, Shock waves and reaction diffusion-equations (2nd ed.), Grundleheren Series 258, Springer-Verlag, New York, 1994.

10. H. Nessyahu and E. Tadmor, Non-oscillatory central differencing for hyperbolic conservation laws, J Comp Phys 87 (1990), 408-463.

11. X.-D. Liu and E. Tadmor, Third order nonoscillatory central scheme for hyperbolic conservation laws, Numerische Mathematik 79 (1998), 397-425.

12. G.-S. Jiang and E. Tadmor, Non-oscillatory central schemes for multidimensional hyperbolic conservation laws, SIAM J Sci Comp 19 (1998), 1892-1917.

13. B. Cockburn, C. Johnson, C.-W. Shu, and E. Tadmor, Advanced Numerical Approximation of Nonlinear Hyperbolic Equations, A. Quarteroni (Ed.), Lecture Notes in Math 1697, Springer, New York, 1997.

14. A. Kurganov and E. Tadmor, New high-resolution central schemes for nonlinear conservation laws and convection-diffusion equations, J Comp Phys 160 (2000), 214-282.

15. A. Kurganov and D. Levy, A third-order semi-discrete central scheme for conservation laws and convection-diffusion equations, SIAM J Sci Comp 22 (2000), 1461-1488.

16. A. Kurganov and G. Petrova, A third-order semi-discrete genuinely multidimensional central scheme for hyperbolic conservation laws and related problems, Numerische Mathematik 88 (2001), 683-729.

17. A. Kurganov, S. Noelle, and G. Petrova, Semi-discrete central-upwind scheme for hyperbolic conservation laws and Hamilton-Jacobi equations, SIAM J Sci Comp 23 (2001), 707-740.

18. A. Kurganov and G. Petrova, Central Schemes and Contact Discontinuities, Math Model Numer Anal 34 (2000), 1259-1275.

19. S. Jin and Z. Xin, The relaxation schemes for systems of conservation laws in arbitrary space dimensions, CPAM 48 (1995), 235-276.

20. X.-D. Liu and S. Osher, Convex ENO high order multi-dimensional schemes without field by field decompositions or staggered grids, J Comput Phys 142 (1998), 304-330. 
21. A. Harten, High resolution schemes for hyperbolic conservation laws, J Comp Phys 49 (1983), 357-393.

22. A. Harten, B. Engquist, S. Osher, and S. R. Chakravarthy, Uniformly high order accurate essentially non-oscillatory schemes III, J Comp Phys 71 (1987), 231-303.

23. A. Kurganov, Conservation laws: stability of numerical approximations and nonlinear regularization, Ph.D. Thesis, Tel-Aviv University, Israel, 1997.

24. S. Osher and E. Tadmor, On the convergence of difference approximations to scalar conservation laws, Math Comp 50 (1988), 19-51.

25. B. van Leer, Towards the ultimate conservative difference scheme, V. A second order sequel to Godunov's method, J Comp Phys 32 (1979), 101-136.

26. D. Levy, G. Puppo, and G. Russo, A third order central WENO scheme for 2D conservation laws, Appl Numer Math 33 (2000), 407-414.

27. D. Levy, G. Puppo, and G. Russo, Compact central WENO schemes for multidimensional conservation laws, SIAM J Sci Comp 22 (2000) 656-672.

28. X.-D. Liu and S. Osher, Nonoscillatory high order accurate self similar maximum principle satisfying shock capturing schemes. I, SIAM J Numer Anal 33 (1996), 760-779.

29. C.-W. Shu and S. Osher, Efficient implementation of essentially non-oscillatory shock-capturing schemes, J Comp Phys 77 (1988), 439-471.

30. C.-W. Shu, Total-variation-diminishing time discretizations, SIAM J Sci Comp 6 (1988), 1073-1084.

31. A. Kurganov and E. Tadmor, New high-resolution semi-discrete central schemes for Hamilton-Jacobi equations, J Comp Phys 160 (2000), 720-742. 